\title{
Co-production as an approach to developing stakeholder partnerships to reduce mental health inequalities: an evaluation of a pilot service
}

\author{
Saumu Lwembe ${ }^{1}$, Stuart A. Green ${ }^{2}$, Jennifer Chigwende ${ }^{3}$, Tom Ojwang ${ }^{4}$ and Ruth Dennis ${ }^{5}$ \\ ${ }^{1}$ Senior Public Health Practitioner, Tri-Borough Public Health Service, London, UK \\ ${ }^{2}$ Public Health Research Fellow, NIHR CLAHRC for North West London, Imperial College London, London, UK \\ ${ }^{3}$ Psychological Wellbeing Practitioner, West London Mental Health NHS Trust, London, UK \\ ${ }^{4}$ Chief Executive, Opportunity for All, London, UK \\ ${ }^{5}$ Clinical Psychologist, West London Mental Health NHS Trust, London, UK
}

\begin{abstract}
Aim: This study aimed to evaluate a pilot cross-sector initiative - bringing together public health, a community group, primary mental health teams and patients - in using co-production approaches to deliver a mental health service to meet the needs of the black and minority ethnic communities. Background: Black and minority ethnic communities continue to face inequalities in mental health service access and provision. They are underrepresented in low-level interventions as they are less likely to be referred, and more likely to disengage from mainstream mental health services. Effective models that lead to improved access and better outcomes are yet to be established. It has long been recognised that to be effective, services need to be more culturally competent, which may be achieved through a co-production approach. Methods: This study aimed to evaluate the role of co-production in the development of a novel community mental health service for black and minority ethnic service users. Qualitative research methods, including semi-structured interviews and focus groups, were used to collect data to examine the use of co-production methods in designing and delivering an improved mental health service. Findings: Twenty-five patients enrolled into the study; of these, 10 were signposted for more intensive psychological support. A 75\% retention rate was recorded (higher than is generally the case for black and minority ethnic service users). Early indications are that the project has helped overcome barriers to accessing mental health services. Although small scale, this study highlights an alternative model that, if explored and developed further, could lead to delivery of patient-centred services to improve access and patient experience within mental health services, particularly for black and minority ethnic communities.
\end{abstract}

Key words: anxiety; BME mental health; coproduction; ethnicity; health inequalities; minority groups; psychotherapy

Received 2 October 2015; revised 28 December 2015; accepted 29 March 2016;

first published online 2 May 2016

\section{Introduction}

\section{Ethnicity and mental health}

Compared to the white majority, black and minority ethnic (BME) people in England continue to experience inequalities in mental health outcomes with inequities in access and provision of appropriate services (Palmer and Ward, 2007), (Street et al., 2005). Set within a wider context of cross-cultural stigma around

Correspondence to: Saumu Lwembe, Senior Public Health Practitioner, Tri-Borough Public Health Service (City of Westminster, London Borough of Hammersmith and Fulham, Royal borough of Kensington and Chelsea), Westminster City Hall, 64 Victoria Street, London SW1E 6QP, London, UK. Email: slwembe@westminster.gov.uk 
mental health problems, they are said to face entrenched difficulties both within their communities and in their relationships with services (Wood et al., 2011). Fitch et al. (2010) identified four key barriers that BME people face in relation to accessing mental health services: socio-cultural difficulties (health beliefs and mistrust of services); systemic problems (lack of culturally competent practices in mental health services); economic issues; and individual barriers (denial of mental health problems).

Studies indicate that black men are almost twice as likely as white men to be detained in police custody under Section 136 of the Mental Health Act (Docking et al., 2009) and are 40-60\% more likely than average population to be admitted to hospital from a criminal justice referral (Care Quality Commission, 2011). A 2004 study by Rethink found that $88 \%$ of BME service users had been forcibly restrained under a section of the Mental Health Act compared to $43 \%$ of white service users; in community settings $\mathrm{BME}$ individuals were $40 \%$ more likely to be turned away when they asked for help than their white counterparts (Rethink, 2004). Audini et al. (2002) concluded that black people are six times more likely to be detained under the Mental Health Act Part II than white people. Inadvertently, within BME groups these factors create a high level of fear associated with mental health treatment; that they will receive inappropriate and poor treatment (eg, excessive restraint and medication) and be discriminated against' [Department of Health (DoH), 2004: 6]. Resultantly, self-initiated help among BME people is low: they are less likely to access care through a general practitioner (Morgan et al., 2005) or be referred to mental health services (Fernando and Fernando, 2003); are under-represented in primary mental health services (Boneham et al., 1997); more likely to disengage from mainstream mental health services (McKenzie, 2012); and have worse mental health outcomes (Palmer and Ward, 2007).

\section{Improving access and improving outcomes for psychological therapies}

Considerable effort has gone into widening access to mental health services by BME people. In 2005, the DoH published an action plan to improve equality of access, experience and outcomes for BME mental health service users (DoH, 2005). The DoH initiated the 'Improving Access to Psychological Therapies (IAPT) programme aimed to address health inequalities in mental health services through widening access to, and choice of, talking therapies throughout England for people with common mental health disorders including anxiety and depression (CSIP Choice and Access Team, 2007). Analysis of access data in the first year of the IAPT programme identified BME groups as being under-represented within the service (Gyani et al., 2013). As such, a further policy response was initiated: the development of the BME Positive Practice Guide in 2009 to be used by IAPT services to increase reach to these groups (CSIP Choice and Access Team, 2009). Subsequently, a range of models for engaging with BME people in mental health services such as staffing services with practitioners from BME background, the provisions of translation and interpretation services coupled with training of both staff and community providers have been implemented over the years (Moffat et al., 2009; Latif, 2010). Despite this, studies have shown that equity in access and provision can be improved further (Green et al., 2013).

\section{A co-production approach to improving outcomes}

Within many metropolitan areas of the UK there exists a wide range of primary and secondary mental health services. In the locality within which this study was undertaken, IAPT service was initiated in 2009. The IAPT service within the locality is delivered through a partnership between three organisations: a secondary care provider, a primary care provider and a voluntary organisation. Patients can access the service either through self-referrals via email, online forms or a telephone helpline or through their General Practitioner (GP). Following referral, it is usual for an initial assessment to be conducted by telephone, although sometimes this may be done face-to-face, to determine the next course of action depending on severity of their condition. The service has been proactive in engaging people from BME communities, using a range of strategies including employing people from similar ethnicity, promoting services at events aimed at BME people and Primary Health Care Research \& Development 2017; 18: 14-23 
co-locating workers in GP surgeries to increase visibility, however, the uptake among the BME population remains low.

To redress this scenario, a cross-sector initiative was established in 2012 tasked with addressing the inequalities in access to and outcomes of mental health services in the locality. The multi-stakeholder initiative brought together agencies including public health, a community organisation, mental health practitioners and the patients themselves to co-design and co-deliver IAPT services to BME communities. The collaboration was developed using the principles of co-production, an approach that prides in viewing service users as active asset-holders rather than passive consumers; promoting collaborative rather than paternalistic relationship between staff and service users and; putting the focus on delivery of outcomes rather than just services (Morgan and Ziglio, 2007; Foot and Hopkins, 2010). The following framework was agreed by all stakeholders: (a) each partner would have a distinct role in the intervention, but operational decisions to be agreed before implementation. Public Health would lead in evaluation, IAPT - provide psychological services through facilitating group sessions and providing one-one advice to patients, Opportunity for All (a community group) - host the project, train and engage local residents as Mental Health Champions/outreach workers to encourage patients to self-refer to project (b) the service would be delivered at a community setting, co-facilitated by a psychological well-being practitioner (IAPT) and an expert patient (local resident from a BME background), and (c) patients would self-refer, or referred by GPs, IAPT or local residents. Patients admitted to receive the psychological service would have to be (a) from a BME background (classified as non-white according to the Office for National Statistics), (b) resident within the locality of the service, (c) experiencing mild-moderate mental health problems, and (d) aged 18 years or above. The aims of the initiative, as agreed by all stakeholders, were to: recruit local residents residing within the study site to be trained and engaged as Mental Health Champions to undertake targeted outreach within the local community; deliver 2-hourly psychological interventions linked with mild-moderate physical activities over six weeks - delivered by mental health practitioners and co-facilitated by an expert patient; and on-going monitoring and evaluation of

Primary Health Care Research \& Development 2017; 18: 14-23 the project. The engagement of local residents was preferred as it was deemed fit within the principles of coproduction and that the residents were familiar with the study context.

This study was undertaken to evaluate the use of a co-production approach in delivering IAPT services to BME people through the collection and analysis of data generated from interviews with stakeholders that were involved in both delivering and receiving services. The main objective was to assess stakeholder experience in both the participation of the co-production process and their views on barriers and enablers to accessing and completing treatment.

\section{Methods}

\section{Study design}

The study used a qualitative research design consisting of semi-structured interviews and a focus group.

\section{Characteristics of the study site}

The service was delivered in a densely populated borough in West London which has a population of $\sim 180000$ with some areas being within the top $10 \%$ most deprived areas in England (Smith, 2012). A locality of $\sim 2000$ residents with a high proportion of BME people (33\%-white British, $22 \%$ African and $14 \%$ Caribbean and a high proportion of people under the age of 15 at 23\% (Smith, 2012) was selected. The population segmentation in the locality has been described as either 'deprived families in public housing' or 'poorer minority families' (Experian, 2009). Higher levels of deprivation have been associated with higher burden of psychiatric conditions (Rai et al., 2010). Inequalities are observed in ethnicity of patients admitted to adult psychiatric inpatient services within the locality; while the admission rate for patients from white ethnic groups is $58 \%$ times lower than the England average, the admission rate for BME patients is $96 \%$ higher than the England average (London Health Observatory, 2011).

\section{Participants}

Only participants involved in the pilot co-production initiative were included in the study. Twenty-five patients self-referred to the service; of these 10 were signposted for more intensive 
Table 1 Outline of data collection methods and participants

\begin{tabular}{lll}
\hline Data collection method & Stakeholder groups & Participants $(n)$ \\
\hline Interview & Patients (P) & 6 \\
& Community group (CG) & 1 \\
& Public health (PH) & 1 \\
Focus group & Mental health provider (MHP) & 1 \\
& Mental health champion (MHC) & 3 \\
& Patients (P) & 6 \\
\hline
\end{tabular}

psychological support due to the severity of their symptoms, and the remainder were treated within the pilot service, of which 11 completed treatment, representing a retention rate higher than is generally seen in BME service users. Other participants included members of the community group, public health, the mental health provider and Mental Health Champions.

\section{Data collection}

Participatory research methodologies were used in collecting qualitative data. The lead researcher was embedded in the initiative as a participant observer. Semi-structured interviews and a focus group were conducted across a range of stakeholders (Table 1). Semi-structured interviews were conducted with patients $(n=6)$, community group $(n=1)$, public health $(n=1)$, mental health provider $(n=1)$ and mental health champions $(n=3)$. Interviews with patients were conducted at entry to the service and exit. A single focus group was held with patients $(n=6)$ following completion of treatment.

\section{Analysis}

Hand-written field notes and audio recordings were made during the semi-structured interviews. Focus group discussions were audio recorded. The recordings were transcribed by the lead researcher (S.L.), and triangulated with field notes. The transcribed information was then sent back to respondents for validation before inclusion in the analysis. All data were analysed using an emergent thematic framework analysis approach (Gale et al., 2013).

\section{Results}

\section{Participation in the co-production process}

Many patients had friends or relatives who had negative experiences within mental health care system and were quite suspicious of mental health professionals. Suspicion was particularly evident during the initial assessment session. Reactions ranged from refusal to sign-up, to overt expressions of disapproval of the mental health team's presence in the at the community site.

'Can you tell us the real reason you are here? You come disguised to help, but we all know what you are after...'.

'Why do you have to come here to deliver your service?'

This prevented the IAPT workers from establishing a therapeutic relationship at the outset, making it difficult for them to implement the community protocol designed and used by the service.

'Many of them also had friends or relatives who had been in the secondary care system so were quite suspicious of mental health professionals. This was initially a barrier that prevented us from establishing a therapeutic relationship'.

(MHP)

Subsequent one-to-one sessions (and transparency imparted by the co-production process) eventually changed the patient's attitudes enabling them to address individual concern in relation to being part of the group and enabled IAPT workers to develop a personalised assessment system for each patient.

'It was interesting to see at the [assessment], where everyone was like 'no not me'... everyone averting their eyes to avoid you asking them questions...to people come in, to have a seat outside waiting, wanting to come

Primary Health Care Research \& Development 2017; 18: 14-23 
in to have one-to-ones and then opening up ... a lot'.

(MHP)

Interviews and focus groups discussions demonstrated respondents across all categories (patients, community group, public health and IAPT) had a high regard for the co-production approach, perceiving it as one of the key factors that enabled participation in and success of the psychological sessions. All respondents reported a positive experience in participation in the co-production process. Many of them felt that by involving patients in deciding the venue, dates, times and influencing the content of the psychological sessions, patients were able to feel more in control and sessions were personalised to their individual needs. As some patients noted:

'...these people are not here to tell me what to do... They are working with me, helping me to realise the greatness within me, to be able to find the strength that I had lost.... They are listening to my needs... [Though] it's a group it feels the session is just for me...'.

(P3, 4, 5, 6 [Focus Group])

The patients felt the process was empowering for them as it helped them to play an active role in the delivery of the service. This view was echoed by service providers. Since they felt ownership of the group sessions, patients were very enthusiastic to complete the sessions, as demonstrated by the high completion rate $(75 \%)$. The enthusiasm and commitment was evidenced by carrying out individual activities at home in between weekly sessions.

'Because I think some people came asking 'so what can you do for me, tell me what you can do for me'... I said...it's not really about what I can do for you; it's about what you would like. 'What? I have never been asked that question by a clinician before', they said. That was really good and helpful and that helped them I think and most of all to tease out the needs of individuals in a group setting'.

(MHP)

'That's what happened in the one to ones they begun to realise that 'oh, we are shaping this. We can say what we want. It's ours'. 'And that was really helpful'.

(MHP)

The co-production process in this project provided invaluable insight to IAPT service, enabling the service to adjust their ways of working to better suit the needs of the patient group. This flexibility contributed to good patient experience, as demonstrated by the attendance and completion of treatment.

'...Back on Track Service [IAPT Service] has standard protocol and guidelines to be followed in delivering group sessions. We realised early on that this was not going to work. The patients instead decided the nature of content they needed to be covered in each session, which was determined by their individual goals they wanted to achieve. Likewise, the Back on track sessions tend to be continuous, each building on the previous session, such that if one fails to attend a subsequent session, they would find it hard to catch-up in future sessions. This didn't seem to work in this intervention. Each session was stand-alone, such that one would not miss anything should they fail to attend a session for whatever reason...'.

(MHP)

\section{Enablers to accessing and completing treatment}

Respondents were asked to identify the factors that enabled them to access or complete treatment. All of the respondents said that by involving patients in developing personal goals, deciding venue, dates, times and session content gave the patients a sense of control. Patients interviewed expressed their commitment to attend the sessions as they felt their goals could only be complete if they attended all the sessions.

'This is our programme. If we fail to come back, we will not achieve what we want. I am stepping towards liveliness; I therefore have to come back'.

Another element of the co-production process that was highly regarded, particularly by the patients, was the engagement of an expert patient from the local community in co-facilitating 
sessions. The expert patient was someone the members of the community, including the patients, held in good esteem, as they were actively involved in community work. The expert patient was perceived as a 'high-achiever' with everything 'going well for them' and was seen as a 'welcome shock' to the patients to then discover that this person also experienced mental health issues.

'It is comforting to know that anyone can suffer from mental health problems...'

The patients were able to relate to the expertpatient's experience with many of them feeling confident that they too can get better.

'Sitting in the same room with [the expert patient] is very inspiring. If [the expert patient] was able to overcome [their illness] I too will be able to...'.

The holistic approach adopted by IAPT was another element cited as an enabler to accessing and completing treatment. Respondents explained how they had other pressing concerns that affected their attendance or concentration in the sessions. For example, many had debt or child-care issues, which they felt were more pressing than mental health problems and needed to be addressed before or as they took part in the sessions, even though these very issues may have contributed to their mental health problems. When they revealed these to the IAPT team, they were offered signposting to appropriate organisations. This turned out to be a good incentive for patients to return to the sessions as they felt their needs were being met.

'We had other people that needed other services that were not psychological. Because we tend to know all these services and have good links with them...community links was key in sustainability [sic]...it's easy for someone to relapse if the key stressors that led them to the mental health experience is not addressed. One patient said to me 'So you tell me how you can help me with breathing. Yesterday, someone came to my door and put a gun to my son's face and said you need to give me this much money or I am going to kill your son'... And I said,
'Breathing is not going to help you. The police will'.

(MHP)

The visible onward support offered to other patients that were not eligible to enrol in the group was seen by the local community as a genuine desire for IAPT to help people.

'There are some people who came and were not able to be part of this intervention but have taken up our services, because they were severely unwell. For example someone who was brought here, the person never leaves their house, very anxious, and needed further Step-3 work with one of our psychologists within our service. It's likely we still have to do home visits with that patient because the patient never leaves the house, so our service will offer that. Another patient had a severe long-term health condition, we allowed the patient to come to the group, but also arranged for counselling to get one to one space to talk about her experiences'.

(MHP)

Patients interviewed cited the friendly nature of IAPT providers, who were also highly culturally competent, as a factor that helped them disclose their personal experiences and continued to attend the sessions.

'She is so friendly. I was able to open up. If it wasn't for her, I wouldn't have come back'.

Delivering the intervention in the community setting, and having face to face assessment was said to be more desirable. All respondents said that they would have found it difficult to pick up the phone or travel to Hammersmith to access the service.

\section{Barriers: attitudes towards mental health}

In this project, many participants perceived the term 'mental health' to mean severe mental illnesses like psychosis or dementia. Common expressions from participants were:

'...I am not mental...like my neighbour...so have not seen the need to access the mental health services...'.

Primary Health Care Research \& Development 2017; 18: 14-23 
'...I need help to drag my neighbour to this project because... has lost it up here [pointing at the head]...'.

'...I was frankly shocked at the amount of stigma around mental health - because we work in mental health and are used to certain terms, and because we know where we are coming from, we assumed that people will at least be aware of certain things. It was shocking to discover that people's knowledge of mental health was of people being 'sectioned' and thinking that such are the people that will be coming to participate in the group intervention. Hence people's initial response was that of fear and mistrust'.

(MHP)

Fear towards the notion of mental illness was further evidenced in participants' refusal at the initial assessment session to fill in registration forms giving their personal details for fear of a documented 'mental health record', with attendant concerns:

'... label me as 'mental' hence unfit to work, which would make it difficult for me to obtain jobs in the future...'.

These attitudes might contribute to the low uptake of mental health services among this population group. By involving the local community from the inception stage, it became possible to capture this view at an early stage. The local community recommended that one should abstain from using the term mental health when promoting the service. To this end, the project title was changed from mental health to 'A step to liveliness', perceived as being both desirable and necessary in capturing what patients would hope to achieve by enrolling into the initiative.

A notable factor recorded in the initial assessment session with the Mental Health Champions was that some of them were in-fact experiencing mildmoderate mental health problems themselves, but appeared oblivious to this. As such, deploying expertise of culturally competent psychological wellbeing practitioners was invaluable. In separate meetings, they were supported in becoming aware of their own needs and took up other support services.
At the initial assessment session, some patients appeared to exhibit obvious forms of 'disengagement' or 'disinterest', this was explored in the subsequent one-one sessions with the therapist. It emerged that some of the participants were in-fact next door neighbours. This made them hesitant to participate in group sessions or disclose their personal experiences for fear of being stigmatised or considered vulnerable. Over time, the realisation that they all have common experiences (including mental health problems) became a common bond.

'Initially, I was scared of what my neighbours would think of me. Then I came to understand that they were also having same problems as me. I felt a sense of relief. I started having hope that they would in fact understand what i was going through'.

\section{Discussion}

The findings of this study concur with other studies regarding mental health work with BME people (Hatzidimitriadou and Keating, 2012). While social and professional attitudes towards mental health patients and treatments have improved over the previous half a century, some minority ethnic groups continue to misunderstand, repel and be frightened by the notion of mental illness, viewing it as encapsulating psychiatric conditions that lead to hospitalisation (Rabkin, 1974). Anthropological studies have posited that the way people from different cultures uphold explanatory models around mental distress will influence their presentation of mental disorder and determine their health-seeking behaviour (Kleinman, 1987; Helman, 2007).

In this project, it was found that stigma and fear of disclosure, were acting in conjunction with an evident suspicion of the service during the initial assessment session, to provide a sizable barrier to engagement. For a community suspicious of mental health services and with complex stereotypes and stigma around mental illnesses, co-production offers a way to break down historical, systemic and socio-cultural barriers and provide better services to those most in need. Boyle et al. (2010) argue that the underlying 
principle of co-production is essentially a commitment to a transformational approach to delivering services, one that assumes that people's needs are better met when they are involved in an equal and reciprocal relationship with professionals, working together to get things done. The transparency imparted by the co-production process in this pilot changed the patient's attitudes and enabled IAPT workers to develop a personalised assessment system for each patient.

The employment of a diverse ethnic workforce is a strategy commonly cited as a key to enabling access to mental health services by BME people as it increases positive response from service users (Increasing Access to Psychological Therapies, 2009), and although the involvement of service users in the planning and delivery of mental health services is generally viewed positively in increasing service satisfaction (Simpson and House, 2002), these strategies had not led to increased uptake of service by BME people within the study site. However, coupling these strategies within the ethos of the co-production approach (which gave the users a say in deciding the MHP and the expert patient); it became apparent that these strategies were highly valued by patients and service providers, resulting in a service with a positive patient experience and good completion rate. The role the expert patient played in building links between the patient group and service providers should not be overlooked.

\section{Strengths and limitations of the study}

By using in-depth qualitative research methods, meaningful insights to the barriers and enablers in accessing services by BME people were attained. The involvement of a participant researcher (S.L.) allowed the development of trust and rapport between service users, IAPT workers and patients; allowing the researcher to explore study topics candidly, something that an outside observer would not have achieved.

The ideology of co-production was consistent throughout all aspects of the study, with all partners involved in delivering the project gathering information on how to better engage with and deliver mental health services for BME communities. The participants found this method acceptable.
The qualitative data capture minimised burden on participants in its design, as the interviews conducted at the entry and exit were embedded as part of the routinely collected data, hence no additional burden was placed on the delivery team.

As a pilot service, the numbers referred to in this study are small and the intervention period is relatively short, but the benefit of a predominantly qualitative study is on the depth of understanding that can be built upon. However, this comes at a price of reduced generalisation. Further work will be required to determine whether results obtained in this pilot intervention will be found in other instances, yet the information obtained as a consequence of the interviews and focus groups provides insight into the needs of BME people in accessing mental health services.

\section{Service implications}

Indications are that the project has made inroads to breaking down barriers to accessing mental health services. The service has been approved for continuation and scaled out to neighbouring boroughs. The project site has been adopted by IAPT as a clinical site, with a notable increased service uptake. As further evidence of the success of this work, some patients have been trained and engaged as mental health champions and expertpatient group facilitators, spreading the community benefit of this intervention. Furthermore, a recent study by Green et al. (2015) exploring predictors of outcome has also demonstrated that while many patients do benefit from the treatments offered by the IAPT service, those from deprived backgrounds with more severe disease do worse, suggesting a need for improving the treatment of these patients, for which co-production may offer a potential solution (Green et al., 2015).

\section{Conclusion}

This study indicates an alternative model that could be further developed leading to delivery of patient-centred services to improve access and patient experience within mental health services for BME people. Within the study a number of factors were identified that were important within

Primary Health Care Research \& Development 2017; 18: 14-23 
the initiative such as the role of the expert patient, the cultural competency of the MHP and joint decision-making by the stakeholders. These factors alone are often employed by services to improve engagement with BME groups but fail to achieve much. Within the co-production context these factors are integral to the service and are operationalised from the start, proving that they are indeed effective mechanisms by which improved engagement and outcomes can be achieved, but within a coproduction framework. As such it is recommended that the service be rolled out in other areas with similar demographic attributes.

\section{Acknowledgements}

The authors thank the participants in this study for contributing to this service evaluation and to thank all the patients and the service providers.

Authors' Contributions: S.L. and S.G. participated in the design of the evaluation. S.L. collected the data, transcribed interviews and performed the analysis. S.L., J.C., T.O., and R.D. conceived of the study. S.L. drafted the initial manuscript; all authors read and approved the final manuscript.

\section{Financial Support}

This article presents independent research commissioned by the National Institute for Health Research (NIHR) under the Collaborations for Leadership in Applied Health Research and Care (CLAHRC) programme for North West London.

\section{Conflicts of Interest}

The views expressed in this publication are those of the author(s) and not necessarily those of the NHS, the NIHR or the Department of Health.

\section{Ethical Standards}

Ethics approval was not required for this work as it is part of a service evaluation and improvement project.

\section{References}

Audini, B. and Lelliott, P. 2002: Age, gender and ethnicity of those detained under Part II of the Mental Health Act 1983. British Journal of Psychiatry 180, 222-26.

Primary Health Care Research \& Development 2017; 18: 14-23
Boneham, M., Williams, K., Copeland, J., Mckibbin, P., Wilson, K., Scott, A. and Sounders, P. 1997: Elderly people from ethnic minorities in Liverpool: mental illness, unmet need and barriers to service use. Health \& Social Care 5, 173-80.

Boyle, D., Coote, A., Sherwood, C. and Slay, J. 2010: RIGHT HERE, RIGHT NOW: taking co-production into the mainstream. London: Nesta.

Care Quality Commission. 2011: Count me in 2010. London: Care Quality Commission.

CSIP Choice and Access Team. 2007: Improving access to psychological therapies: positive practice guide. London: CSIP Choice and Access Team.

CSIP Choice and Access Team. 2009: Black and Minority Ethnic (BME): positive practice guide. London: CSIP Choice and Access Team.

Department of Health (DoH). 2004: Choosing health: making healthy choices easier. London: DoH.

Department of Health (DoH). 2005: Delivering race equality in mental health care. London: DoH.

Docking, M., Grace, K. and Bucke, T. 2009: Police Custody as a 'Place of Safety': Examining the Use of Section 136 of the Mental Health Act 1983.

Experian. 2009: Mosaic: the consumer classification of the United Kingdom. London: Experian.

Fernando, D.S. and Fernando, S. 2003: Cultural diversity, mental health and psychiatry: the struggle against racism. Brunner - Routledge, East Sussex: Routledge.

Fitch, C., Wilson, M. and Worral, A. 2010: Improving in-patient mental health services for Black and minority ethnic patients. London: Royal College of Psychiatrists.

Foot, J. and Hopkins, T. 2010: A glass half-full: how an asset approach can improve community health and well-being. London: Improvement and development agency, Local Government Association.

Gale, N.K., Heath, G., Cameron, E., Rashid, S. and Redwood, S. 2013: Using the framework method for the analysis of qualitative data in multi-disciplinary health research. BMC Medical Research Methodology 13, 117.

Green, S.A., Poots, A.J., Marcano-Belisario, J., Samarasundera, E., Green, J., Honeybourne, E. and Barnes, R. 2013: Mapping mental health service access: achieving equity through quality improvement. Journal of Public Health 35, 286-92.

Green, S.A., Honeybourne, E., Chalkleyl, S.R., Poots, A.J., Woodcock, T., Price, G., Bell, D. and Green, J. 2015: A retrospective observational analysis to identify patient and treatment-related predictors of outcomes in a community mental health programme. BMJ Open 5, e006103.

Gyani, A., Shafran, R., Layard, R. and Clark, D.M. 2013: Enhancing recovery rates in IAPT services: lessons from analysis of the year one data. Behaviour Research and Therapy 51, 597-606.

Hatzidimitriadou, E. and Keating, F. 2012: Evaluation of co-production processes in a community-based mental health project, November, Wandsworth. 
Helman, D.C. 2007: Culture, health and illness, fifth edition. London: Hodder Arnold.

Increasing Access to Psychological Therapies. 2009: Black and Minority Ethnic (BME) Positive Practice Guide. London: Increasing Access to Psychological Therapies.

Kleinman, A. 1987: Anthropology and psychiatry. The role of culture in cross-cultural research on illness. The British Journal of Psychiatry: The Journal of Mental Science 151, 447-54.

Latif, S. 2010: Effective methods of engaging black and minority ethnic communities within health care settings. London: Race Equality Foundation.

London Health Observatory. 2011: London adult mental health scorecard. London: London Health Observatory.

McKenzie, K. 2012: Suicide studies in ethnic minorities: improving the science to help develop policy. Ethnicity \& Health 17, 7-11.

Moffat, J., Sass, B., Mckenzie, K. and Bhui, K. 2009: Improving pathways into mental health care for black and ethnic minority groups: a systematic review of the grey literature. International Review of Psychiatry (Abingdon, England) 21, 439-49.

Morgan, A. and Ziglio, E. 2007: Revitalising the evidence base for public health: an assets model. Promotion \& Education 14 (Suppl), 17-22.

Morgan, C., Mallet, R., Hutchinson, G., Bagalkote, H., Morgan, K., Fearon, P., Dazzan, P., Boydell, J., Mckenzie, K., Harrison, G., Murray, R., Jones, P., Craig, T. and Leff, J. 2005: Pathways to care and ethnicity. 2: source of referral and help-seeking. Report from the AESOP study. The British Journal of Psychiatry: The Journal of Mental Science 186, 290-96.

Palmer, D. and Ward, K. 2007: 'Lost': listening to the voices and mental health needs of forced migrants in London. Medicine, Conflict, and Survival 23, 198-212.

Rabkin, J. 1974: Public attitudes toward mental illness. Schizophrenia Bulletin 1, 9-33.

Rai, D., Skapinakis, P., Wiles, N., Lewis, G. and Araya, R. 2010: Common mental disorders, subthreshold symptoms and disability: longitudinal study. The British Journal of Psychiatry: The Journal of Mental Science 197, 411-12.

Rethink. 2004: Behind closed doors: the current state and future vision of acute mental health care in the UK. London: Rethink.

Simpson, E.L. and House, A.O. (2002) Involving users in the delivery and evaluation of mental health services: systematic review. BMJ 325, 1265-9.

Smith, M. 2012. Prioritising health and wellbeing needs. London: Kensington and Chelsea.

Street, C., Stapelkamp, C. and Taylor, E. 2005: Research into the access and acceptability of services for the mental health of young people from Black and minority ethnic groups. London: Young Minds for Children Mental Health.

Wood, J., Dooher, J., Mclaughlin, B., Yates, S. and Norrie, P. 2011: National evaluation of the community development workers. Leicester: National Mental Health Development Unit. 